\title{
Las tablas de riesgo cardiovascular. Una revisión crítica
}

\author{
A. Álvarez Cosmea \\ Médico Especialista en Medicina de Familia y Comunitaria. \\ C. S. Ventanielles. Oviedo. Asturias
}

\section{RESUMEN}

El riesgo cardiovascular global, definido como la probabilidad de presentar un evento en un periodo determinado, se considera como el mejor método de abordaje de la enfermedad arterioescle rótica; el método de cálculo, a través de las llama das tablas de riesgo cardiovascular (método cuan titativo), ha sido muy divulgado a raíz del estudio de Framingham, base de casi todas ellas.

Las Sociedades Científicas en sus recomendaciones para la prevención de la enfermedad coro naria utilizan estas tablas para identificar a los pa cientes de alto riesgo, prioritarios a la hora de intervenir con fármacos sobre los distintos factores de riesgo; sin embargo existen distintas tablas y existe la controversia de si son válidas al aplicar las en otro tipo de poblaciones de riesgo coronario bajo, como la española; en este artículo se preten de describir las tablas de riesgo más importantes, variables que utilizan, ventajas e inconvenientes, así como su aplicación en la práctica clínica.

Palabras clave: Enfermedad cardiovascular. Factores de riesgo. Predicción. Riesgo cardiovas cular.
The cardiovascular risk charts. $A$ critical review

The global cardiovascular risk, defined as the probability of origin a new event in a definite pe riod of time, is considered the best approaching method to the coronary heart disease; the calcula ting method, through the cardiovascular risk charts (quantitative method), has been very disclosed as a result of the Framingham research, basis of al most all of them.

The scientific societies in its recomendations for the coronary heart disease prevention, use the car diovascular risk charts to identify high risk pa tients, which are priority at the moment of taking part over the different risk factors with drugs; however, there are different chart, and there is a controversy about if them are valid for population with low cardiovascular risk, as the Spanish; in this article, we intend to describe the most impor tant risk charts, used variables, advantages and in conveniences, as well as its application in the clini cal practice.

Key words: Cardiovascular disease. Risk fac tors. Prediction. Cardiovascular risk.

\section{INTRODUCCIÓN}

La enfermedad cardiovascular, al igual que en el resto de países occidentales, es la primera causa de muerte en España (40\% de todas las defunciones en
1992) ${ }^{1}$; las distintas Sociedades científicas en su afán de prevenir la arterioesclerosis, causa fundamental de la enfermedad cardiovascular, y dado su origen multifactorial, recomiendan la estimación del riesgo cardiovascular global para clasificar a las 
personas en los distintos grupos de riesgo, en base a poder priorizar las intervenciones con fármacos sobre los factores de riesgo; así las sociedades europeas $^{2}$ recomiendan la intervención, en orden de mayor a menor de:

- Pacientes con enfermedad coronaria establecida u otras enfermedades ateroescleróticas.

- Personas sanas con riesgo alto de desarrollar enfermedad coronaria u otra enfermedad ateroesclerótica, ya que presentan una combinación de factores de riesgo o un solo factor de riesgo en forma grave.

-Familiares de primer grado de pacientes con enfermedad coronaria de aparición precoz u otra enfermedad ateroesclerótica y de personas sanas con riesgo cardiovascular muy alto.

- Otras personas a las que se accede en la práctica clínica habitual.

El riesgo coronario y/o cardiovascular (RCV) es la probabilidad de presentar una enfermedad coronaria o cardiovascular en un periodo de tiempo determinado, generalmente de 5 ó 10 años; en general, se habla de riesgo coronario o cardiovascular indistintamente ya que ambas medidas se correlacionan bien, aunque algunos autores consideran que multiplicando el riesgo coronario por $4 / 3$ obtenemos una mejor estimación del riesgo cardiovascular'.

Existen dos métodos de cálculo de RCV: cualitativos y cuantitativos; los cualitativos se basan en la suma de factores de riesgo y clasifican al individuo en riesgo leve, moderado y alto riesgo; los cuantitativos nos dan un número que es la probabilidad de presentar un evento cardiovascular en un determinado tiempo; la forma de cálculo es a través de programas informáticos, basados en ecua- ciones de predicción de riesgo, o las llamadas ta blas de riesgo cardiovascular, que ahora pasaremos a describir.

La estimación del riesgo cardiovascular global por este método tiene 3 objetivos clínicos fundamentales:

- Identificar pacientes de alto riesgo que precisan atención e intervención inmediata.

- Motivar a los pacientes para que sigan el tratamiento y así reducir riesgo.

- Modificar la intensidad de la reducción de riesgo en base al riesgo global estimado ${ }^{4}$.

El objetivo fundamental es clasificar a los pacientes e intervenir con fármacos en individuos de alto riesgo, que se define según criterio de las distintas sociedades y organismos, como refleja la tabla I.

Las tablas de riesgo cardiovascular más utilizadas están basadas en la ecuación de riesgo del estudio de Framingham ${ }^{5}$; describiremos las más importantes que son: Framingham clásica, Framingham por Categorías, nuevas tablas de Framingham, Sociedades Europeas, Sociedades Británicas, Nueva Zelanda y Sheffield.

\section{TABLAS DE RIESGO DE FRAMINGHAM}

Utiliza un método de puntuación en base a las siguientes variables: edad (35-74 años), sexo, HDLcolesterol, colesterol total, presión arterial sistólica, tabaquismo (sí/no), diabetes (sí/no) e hipertrofia ventricular izda. (HVI) (sí/no) (Fig. 1); con ello podemos calcular el riesgo coronario a los 10 años que incluye: angina estable, infarto de miocardio (IAM) y muerte coronaria.

Tabla I

CRITERIOS DE ALTO RIESGO SEGÚN RECOMENDACIONES DE LAS DISTINTAS SOCIEDADES Y ORGANISMOS

\begin{tabular}{lll}
\hline Sociedades & Tabla riesgo & Alto riesgo \\
\hline PAPPS-semFYC & Framingham clásica & $\geq 20 \% 10$ años \\
\hline S. Europeas & S. Europeas & $\geq 20 \% 10$ años \\
\hline S. Británicas & S. Británicas & $\geq 30 \% 10$ años \\
\hline Nueva Zelanda & Nueva Zelanda & $\geq 10-15 \% 5$ años \\
& Sheffield & $\geq 30 \% 10$ años
\end{tabular}

PAPPS-semFYC: Programa de Actividades Preventivas y Promoción de la Salud de la Sociedad de Medicina y Comunitaria; S. Europeas: Sociedades Europeas de Cardiología, Hipertensión Arterial y Arteriosclerosis; S. Británica: Sociedades Británicas de Cardiología, Hipertensión, Lípidos y Diabetes; Nueva Zelanda: Ministerio de Salud Pública de Nueva Zelanda. 


\begin{tabular}{|c|c|c|c|c|c|c|c|c|c|}
\hline $\begin{array}{l}\text { Mujer } \\
\text { edad }\end{array}$ & Puntos & $\begin{array}{l}\text { Hombre } \\
\text { edad }\end{array}$ & Puntos & $\begin{array}{l}\text { HDL-c } \\
\mathrm{mg} / \mathrm{dl}\end{array}$ & Puntos & Colest. - total & Puntos & PAS & Puntos \\
\hline 30 & -12 & 30 & -2 & $25-26$ & 7 & $139-151$ & -3 & $98-104$ & -2 \\
\hline 31 & -11 & 31 & -1 & $27-29$ & 6 & $152-166$ & -2 & $105-112$ & -1 \\
\hline 32 & -9 & $32-33$ & 0 & $30-32$ & 5 & $167-182$ & -1 & $113-120$ & 0 \\
\hline 33 & -8 & 34 & 1 & 33-35 & 4 & 183-199 & 0 & $121-129$ & 1 \\
\hline 34 & -6 & $35-36$ & 2 & $36-38$ & 3 & $200-219$ & 1 & $130-139$ & 2 \\
\hline 35 & -5 & $37-38$ & 3 & $39-42$ & 2 & $220-239$ & 2 & $140-149$ & 3 \\
\hline 36 & -4 & 39 & 4 & $43-46$ & 1 & $240-262$ & 3 & $150-160$ & 4 \\
\hline 37 & -3 & $40-41$ & 5 & $47-50$ & 0 & $263-288$ & 4 & 161-172 & 5 \\
\hline 38 & -2 & $42-43$ & 6 & $51-55$ & -1 & $289-315$ & 5 & $173-185$ & 6 \\
\hline 39 & -1 & $44-45$ & 7 & $56-60$ & -2 & $316-330$ & 6 & & \\
\hline 40 & 0 & $46-47$ & 8 & 61-66 & -3 & & & & \\
\hline 41 & 1 & $48-49$ & 9 & $67-73$ & -4 & & & & \\
\hline $42-43$ & 2 & $50-51$ & 10 & $74-80$ & -5 & & & & \\
\hline 44 & 3 & $52-54$ & 11 & $81-87$ & -6 & & & & \\
\hline $45-46$ & 4 & $55-56$ & 12 & $88-96$ & -7 & & & & \\
\hline $47-48$ & 5 & $57-59$ & 13 & & & & & & \\
\hline $49-50$ & 6 & $60-61$ & 14 & & & & & & \\
\hline $51-52$ & 7 & $62-64$ & 15 & & & & & & \\
\hline 53-55 & 8 & $65-67$ & 16 & & & & & & \\
\hline $56-60$ & 9 & $68-70$ & 17 & & & & & & \\
\hline 61-67 & 10 & 71-73 & 18 & & & & & & \\
\hline $68-74$ & 11 & 74 & 19 & & & & & & \\
\hline
\end{tabular}

Puntos y Riesgo coronario a los 10 años

\begin{tabular}{|cccccccc|}
\hline Puntos & Riesgo & Puntos & Riesgo & Puntos & Riesgo & Puntos & Riesgo \\
\hline$<1$ & $<2 \%$ & 9 & $5 \%$ & 17 & $13 \%$ & 25 & $27 \%$ \\
\hline 2 & $2 \%$ & 10 & $6 \%$ & 18 & $14 \%$ & 26 & $29 \%$ \\
\hline 3 & $2 \%$ & 11 & $6 \%$ & 19 & $16 \%$ & 27 & $31 \%$ \\
\hline 4 & $2 \%$ & 12 & $7 \%$ & 20 & $18 \%$ & 28 & $33 \%$ \\
\hline 5 & $3 \%$ & 13 & $8 \%$ & 21 & $19 \%$ & 29 & $36 \%$ \\
\hline 6 & $3 \%$ & 14 & $9 \%$ & 22 & $21 \%$ & 30 & $38 \%$ \\
\hline 7 & $4 \%$ & 15 & $10 \%$ & 23 & $23 \%$ & 31 & $40 \%$ \\
\hline 8 & $4 \%$ & 16 & $12 \%$ & 24 & $25 \%$ & 32 & $42 \%$ \\
\hline
\end{tabular}

\begin{tabular}{|cc|}
\hline Otros Factores & Puntos \\
\hline Tabaquismo & 4 \\
\hline Diabetes: Hombres & 3 \\
Mujeres & 6 \\
\hline Hipertrofia Vizda. & 9 \\
\hline
\end{tabular}


Es el método recomendado por el grupo PAPPSsemFYC ${ }^{6}$, entre otros; tiene como ventajas que podemos asumir si no tenemos datos de HDL-colesterol una cifra de $39 \mathrm{mg} / \mathrm{dl}$ en varones y de $43 \mathrm{mg} / \mathrm{dl}$ en mujeres; además si no tenemos un electrocardiograma $(\mathrm{EKG})$, consideraremos que no presenta HVI, por lo que conociendo su condición de diabetes o no, con datos de su hábito tabáquico, colesterol total y presión arterial sistólica, podríamos realizar un cálculo aproximado de su riesgo cardiovascular, asumiendo lo anteriormente expuesto.

Consideramos como inconvenientes de esta tabla:

—Está basada en el estudio de Framingham, una población americana con una mayor prevalencia y riesgo de enfermedad cardiovascular que la nuestra, y aunque algunos estudios indican que la predicción de riesgo es aceptable en el Norte de Euro$\mathrm{pa}^{7}$, otros creen que sobrestima el riesgo en otras poblaciones, como Reino Unido ${ }^{8}$ o mediterráneas, como Italia ${ }^{9}$ o España ${ }^{10}$, por lo que tendríamos que tener cierta cautela en estos casos.

- Son útiles al comparar poblaciones, pero plantean cierto grado de incertidumbre a la hora de valorar el riesgo individual.

- No considera dentro de sus variables la historia familiar de enfermedad coronaria precoz $^{8,11}$ (se considera antecedente familiar positivo si puede documentarse una enfermedad coronaria clínica o una muerte súbita en un familiar de primer grado menor de 55 años si es hombre o de 65 años si es mujer $)^{14}$.

- Tampoco contempla otros factores de riesgo como triglicéridos (sobre todo si se acompaña de una cifra baja de HDL-colesterol), fibrinógeno, homocisteína, etc. ${ }^{11}$;

- No se puede aplicar en pacientes con una enfermedad cardiovascular manifiesta;

-Predice mejor el riesgo en sujetos de mayor edad que en jóvenes

- No son adecuadas para individuos que presenten un único factor de riesgo (dislipemia, HTA o diabetes) grave o muy grave ${ }^{12}$; en el caso de diabetes, probablemente se infravalore el riesgo ya que hoy en día este se equipara a la prevención secundaria ${ }^{13}$.

\section{TABLAS DE FRAMINGHAM POR CATEGORÍAS}

Con el fin de adaptar la ecuación de riesgo de Framingham a las recomendaciones del National Cholesterol Education Program (NCEP) ${ }^{14}$ y $V$ Joint National Committee (V JNC) ${ }^{15}$, en 1998 se publican las llamadas tablas de riesgo de Framingham por categorías (Wilson $)^{16}$; las variables que utiliza son: edad (30-74 años), sexo, tabaquismo (sí/no), diabetes (sí/no) y las categorías de: HDL-colesterol, colesterol total (existen otras tablas cuya categoría no es el colesterol total, sino el LDL- colesterol) y presión arterial sistólica y diastólica (Fig. 2); esta tabla sirve para calcular la probabilidad de presentar una enfermedad coronaria total (angina estable, inestable, IAM y muerte coronaria), en un periodo de 10 años; posteriormente, en 1999, Grundy ${ }^{17}$ realizó una pequeña modificación de las tablas anteriores, considerando la diabetes como glucosa basal $>126$ $\mathrm{mg} / \mathrm{dl}$ (acorde con los nuevos criterios de la Asociación Americana de Diabetes), además de poder realizar un cálculo del riesgo de presentar lo que ellos llaman "hard $C H D$ " o "eventos duros", que incluye sólo la angina inestable, IAM y muerte coronaria (Fig. 3).

Es el método recomendado en el momento actual por la American Heart Association (AHA) y el American College of Cardiology (ACC). Además no solamente podemos utilizar el riesgo absoluto de enfermedad coronaria total o eventos duros, sino que también podemos ver el riesgo relativo en una tabla reflejada en colores (según riesgo) ${ }^{17}$; este concepto de riesgo relativo tiene una mayor importancia en personas jóvenes y ancianos (ya que en estos el riesgo absoluto aumenta con la edad por el acúmulo natural de aterogénesis) y se define como el cociente entre el riesgo absoluto y el riesgo bajo (definido como aquella persona de la misma edad, con una tensión arterial $<120 / 80 \mathrm{mmHg}$, un colesterol total entre 160-199 mg/dl, un HDL-colesterol $>45 \mathrm{mg} / \mathrm{dl}$, no fumador y no diabético) o el riesgo medio de una determinada población ${ }^{17,18-20 .}$ (Fig. 3a); así, una vez calculados los puntos según la tabla de la figura 3 , nos vamos a la columna de la edad del paciente y la intersección de ambos corresponde a una cuadrícula con un número y color que puede ser verde (riesgo por debajo del medio de la población), violeta (riesgo medio de la población), amarillo (moderadamente por encima del riesgo medio) y rojo (alto riesgo).

Como ventajas, podemos resaltar:

- Se ajusta a las recomendaciones nacionales del NCEP y V JNC, puntuando los factores de riesgo en base a su severidad ("categorías")"

-Utiliza una variable menos, ya que no considera la HVI diagnosticada por EKG.

- Se puede utilizar el concepto de riesgo relativo en una tabla de colores.

-Incluye la probabilidad de "eventos duros", objetivo fundamental en los ensayos clínicos.

Quizás esta última sea la característica más importante de estas tablas, ya que según algunos autores, la exactitud de este sistema por categorías es similar al anterior de variables continuas ${ }^{21}$. 


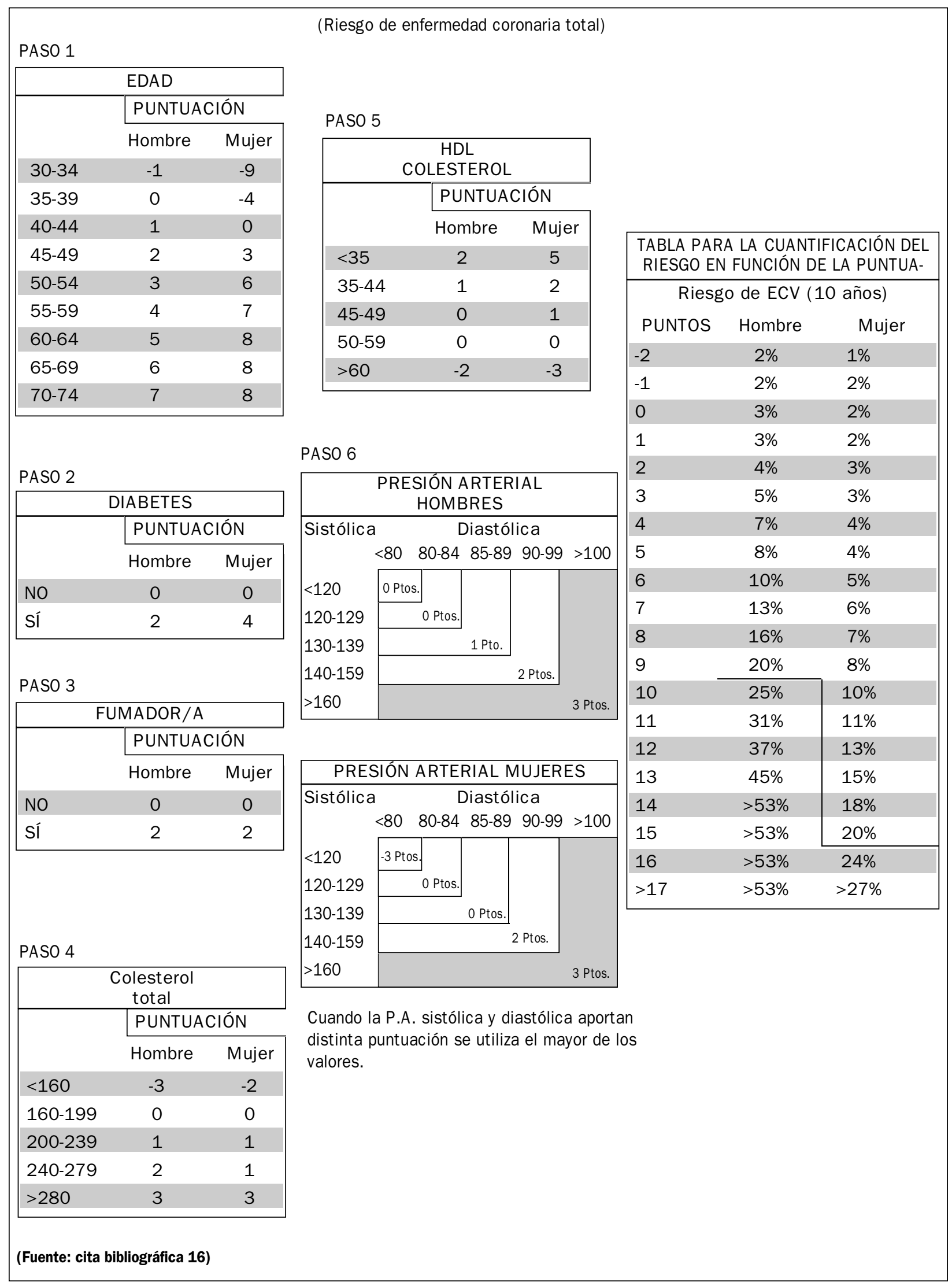

Figura 2

Tablas de riesgo de Framingham por categorías (Wilson), según colesterol total. ECV: enfermedad cardiovascular. 


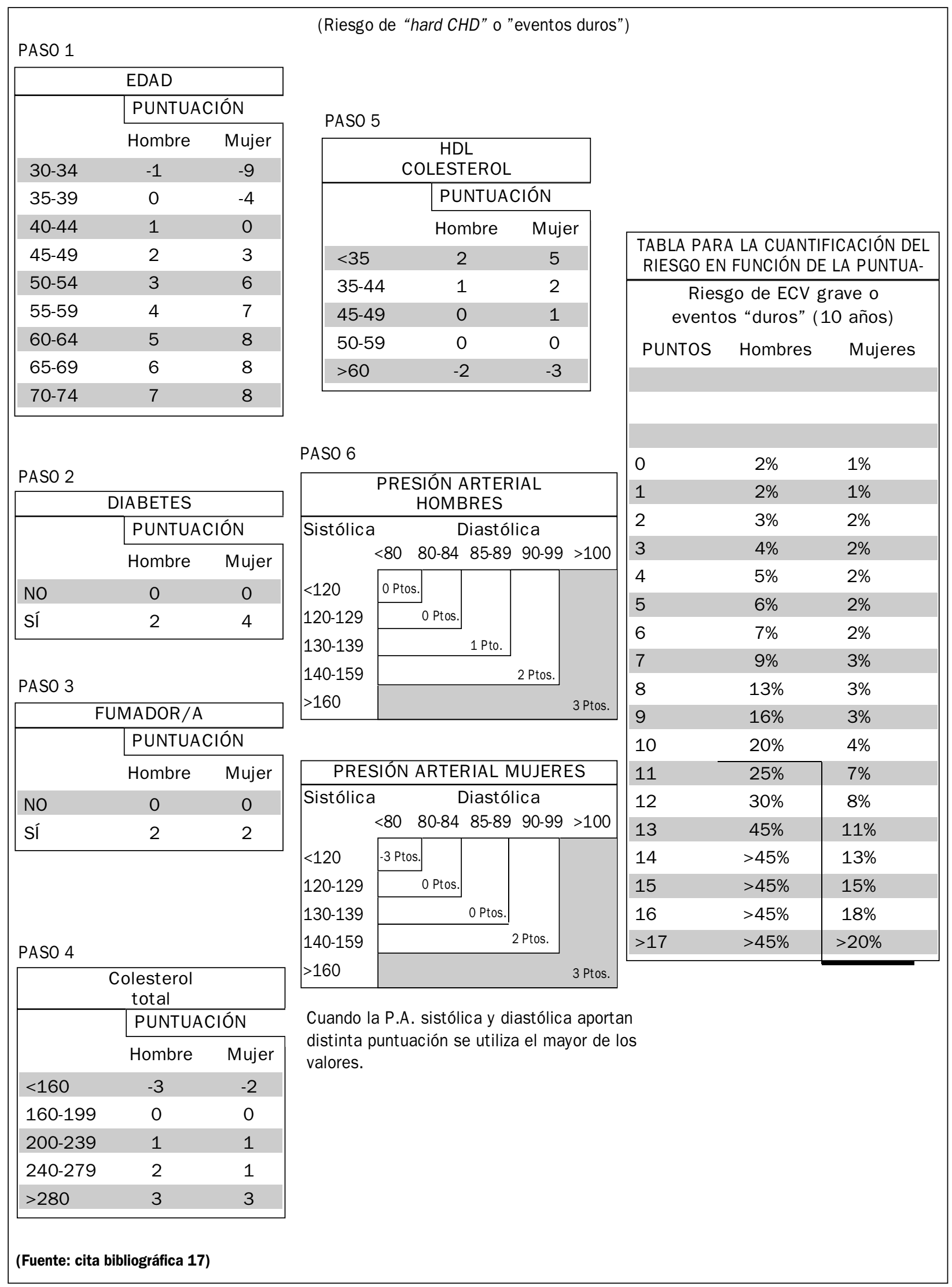

Figura 3

Tablas de riesgo de Framingham por categorías (Grundy). ECV: enfermedad cardiovascular. 


\begin{tabular}{|c|c|c|c|c|c|c|c|c|c|c|c|}
\hline Edad & $30-34$ & $35-39$ & $40-44$ & $45-49$ & $50-54$ & $55-59$ & $60-64$ & $65-69$ & $70-74$ & & \\
\hline $\begin{array}{c}\text { (Bajo nivel } \\
\text { de } \\
\text { riesgo) }\end{array}$ & $(2 \%)$ & $(3 \%)$ & $(3 \%)$ & $(4 \%)$ & $(5 \%)$ & $(7 \%)$ & $(8 \%)$ & $(10 \%)$ & $(13 \%)$ & $\begin{array}{c}\text { Riesgo } \\
\text { absoluto }\end{array}$ & $\begin{array}{c}\text { Riesgo } \\
\text { Absoluto }\end{array}$ \\
\hline $\begin{array}{c}\text { Puntos } \\
\text { T }\end{array}$ & & & & & & & & & & $\begin{array}{c}\text { Total } \\
\text { CHD }\end{array}$ & $\begin{array}{c}\text { Hard } \\
\text { CHD\# }\end{array}$ \\
\hline 0 & 1,0 & & & & & & & & & $2 \%$ & $2 \%$ \\
\hline 1 & 1,5 & 1,0 & 1,0 & & & & & & & $3 \%$ & $2 \%$ \\
\hline 2 & 2,0 & 1,3 & 1,3 & 1,0 & & & & & & $4 \%$ & $3 \%$ \\
\hline 3 & 2,5 & 1,7 & 1,7 & 1,3 & 1,0 & & & & & $5 \%$ & $4 \%$ \\
\hline 4 & 3,5 & 2,3 & 2,3 & 1,8 & 1,4 & 1,0 & & & & $7 \%$ & $5 \%$ \\
\hline 5 & 4,0 & 2,6 & 2,6 & 2,0 & 1,6 & 1,1 & 1,0 & & & $8 \%$ & $6 \%$ \\
\hline 6 & 5,0 & 3,3 & 3,3 & 2,5 & 2,0 & 1,4 & 1,3 & 1,0 & & $10 \%$ & $7 \%$ \\
\hline 7 & 6,5 & 4,3 & 4,3 & 3,3 & 2,6 & 1,9 & 1,6 & 1,3 & 1,0 & $13 \%$ & $9 \%$ \\
\hline 8 & 8,0 & 5,3 & 5,3 & 4,0 & 3,2 & 2,3 & 2,0 & 1,6 & 1,2 & $16 \%$ & $13 \%$ \\
\hline 9 & 10,0 & 6,7 & 6,7 & 5,0 & 4,0 & 2,9 & 2,5 & 2,0 & 1,5 & $20 \%$ & $16 \%$ \\
\hline 10 & 12,5 & 8,3 & 8,3 & 6,3 & 5,0 & 3,6 & 3,1 & 2,5 & 1,9 & $25 \%$ & $20 \%$ \\
\hline 11 & 15,5 & 10,3 & 10,3 & 7,8 & 6,1 & 4,4 & 3,9 & 3,1 & 2,3 & $31 \%$ & $25 \%$ \\
\hline 12 & 18,5 & 12,3 & 12,3 & 9,3 & 7,4 & 5,2 & 4,6 & 3,7 & 2,8 & $37 \%$ & $30 \%$ \\
\hline 13 & 22,5 & 15,0 & 15,0 & 11,3 & 9,0 & 6,4 & 5,6 & 4,5 & 3,5 & $45 \%$ & $35 \%$ \\
\hline$>14$ & 26,5 & $>17,7$ & $>17,7$ & $>13,3$ & $>10,6$ & $>7,6$ & $>6,6$ & $>5,3$ & $>4,1$ & $>53 \%$ & $>45 \%$ \\
\hline
\end{tabular}

\title{
Bajo límite de riesgo $\square \quad$ Límite $\square \quad$ Levemente por encima del límite de riesgo $\square \quad$ Riesgo alto $\square$
}

\begin{abstract}
†Total CHD: Enfermedad coronaria total.
\#Hard CHD: "eventos duros", equivalente a muerte coronaria e infarto de miocardio.

*Bajo nivel de riesgo es aquella persona de la misma edad, con una tensión arterial $<120 / 80 \mathrm{mmHg}$, con colesterol total entre $160-199 \mathrm{mg} / \mathrm{dl}$, un HDL-colesterol $>45 \mathrm{mg} / \mathrm{dl}$, no fumador y no diabético.
\end{abstract}

(Fuente: cita bibliográfica 17)

Figura 3a

Riesgo relativo según tablas de riesgo de Framingham por Categorías (Grundy) (Hombres).

Los inconvenientes son los mismos que los enumerados en el apartado anterior, ya que utiliza las mismas variables, salvo las categorías de lípidos y tensión arterial y que no tiene en cuenta la HVI.

\section{NUEVAS TABLAS DE FRAMINGHAM ( $\left.D^{\prime} A G O S T I N O\right)$}

A medida que se analizan nuevos datos y resultados del estudio de Framingham, se van añadiendo a los modelos matemáticos, nuevos factores de riesgo a tener en cuenta; de este modo se publican en un artículo del American Heart Journal ${ }^{22}$ nuevas tablas de riesgo; utilizan variables distintas según sean hombres o mujeres y existen tablas para prevención primaria (probabilidad de presentar un primer evento) y prevención secundaria (probabilidad de presentar una enfermedad coronaria o accidente cerebrovascular en aquéllos que ya han sufrido un evento cardiovascular).

Se pueden aplicar en pacientes entre 35-74 años y el riesgo se calcula a más corto plazo (2 años); en varones, en prevención primaria, las variables utilizadas son: edad, colesterol total y HDL-colesterol, diabetes (sí/no), tabaco (sí/no), presión arterial sistólica (tratada/no tratada); en el caso de las mujeres existe un modelo que incluye triglicéridos y otro que no; el resto de las variables, además de las mencionadas para los hombres, incluye: edad (con menopausia/sin menopausia), ingesta de alcohol; la puntuación final también es distinta según su situación respecto a la menopausia.

En los casos de prevención secundaria en varones, el cálculo de riesgo de otro evento coronario o accidente cerebrovascular en un periodo de 2 años, 
se realiza en base a la edad, colesterol total, HDLcolesterol y diabetes; en mujeres, además se tiene en cuenta el tabaco y la presión arterial sistólica (Figs. 4 y 5).

Entre las ventajas de estas tabla, destaca: años).

El cálculo de riesgo se hace a corto plazo (2

- Se incluyen otros factores de riesgo, sobre todo en mujeres, como el consumo de alcohol, la menopausia y los triglicéridos.

- Se puede calcular el riesgo también en pacientes en prevención secundaria.

-Utiliza el cociente colesterol total/HDL-colesterol, mejor predictor de enfermedad coronaria.

Entre los inconvenientes los autores resaltan que, al igual que las anteriores, se debe de tener cautela al aplicarlas a otras poblaciones, sobre todo las de bajo riesgo.

\section{TABLAS DE RIESGO DE LAS SOCIEDADES EUROPEAS}

En 1998 se realiza una revisión de las recomendaciones publicadas en 1994 de las Sociedades Europeas de Cardiología, Arterioesclerosis e Hipertensión, respecto a la prevención de la enfermedad coronaria en la práctica clínica, en la que también participan la Sociedad Internacional de Medicina del Comportamiento, Sociedad Europea de Medicina General y Medicina de Familia y la Red Europea del Corazón ${ }^{2}$.

Las tablas que recomiendan para el cálculo de riesgo coronario (definido como angina, IAM y muerte coronaria) en un periodo de 10 años, utilizan las siguientes variables: edad (30-70 años), sexo, tabaco, colesterol total y presión arterial sistólica; existen 2 tipos de tablas, dependiendo si el individuo es o no diabético (Fig. 6); el nivel de riesgo viene representado en la tabla en colores, considerando por consenso un riesgo alto (umbral de tratamiento con fármacos), si alcanza o supera el $20 \%$ a los 10 años, o si supera el $20 \%$ al proyectarlo a la edad de 60 años.

Las tablas se utilizan de la siguiente forma: una vez elegida la tabla en base a su condición de diabetes o no, nos vamos al cuadro correspondiente según edad, sexo y columna según sea fumador o no; después se busca la casillla que más cerca este en base a cifras de presión arterial sistólica y colesterol total; se considera que el riesgo es mayor que el reflejado en las tablas si presenta: hiperlipemia familiar, antecedentes familiares de enfermedad coronaria precoz, HDL-colesterol bajo $(<39 \mathrm{mg} / \mathrm{dl}$ en varones y de $43 \mathrm{mg} / \mathrm{dl}$ en mujeres), triglicéridos mayores de $180 \mathrm{mg} / \mathrm{dl}$ y si el individuo se aproxima a la siguiente categoría de edad.
Además de las Sociedades Europeas, es el método recomendado por la Sociedad Española de Cardiología en su guía de prevención cardiovascular y rehabilitación cardiaca ${ }^{23}$, así como el utilizado en el Consenso del Colesterol en España, 200024.

Entre las ventajas ${ }^{23}$ de este tipo de tablas coloreadas o gráficos podemos destacar: son fáciles de utilizar (sólo cinco variables), permite situar al individuo en relación al resto de la población según código de color, puede servir para negociar con el paciente y para que vea reflejados los beneficios de intervención sobre los factores de riesgo, y en jóvenes ilustra como estará ese paciente dentro de unos años si no corrige los factores que identificamos.

Entre los inconvenientes tenemos que destacar que no tiene en cuenta el HDL-colesterol, ya que según el Documento ${ }^{2}$ no se solicita de forma rutinaria en toda Europa; sin embargo en España los valores elevados de HDL-colesterol, podrían explicar, aunque no de forma exclusiva, las tasas de mortalidad más bajas que en otros países desarrollados ${ }^{25}$; además el HDL-colesterol bajo se considera claramente un factor de riesgo independiente de enfermedad coronaria $^{26}$; por todo esto, llama la atención que estas tablas de riesgo fueran las recomendadas para el cálculo de riesgo cardiovascular en el Consenso de la Colesterolemia en España, 2000.

\section{TABLAS DE LAS SOCIEDADES BRITÁNICAS}

En 1998 las Sociedades Británicas de Hipertensión, Diabetes, Lípidos y Cardiología publican también, 2 meses después de las europeas, sus recomendaciones para la prevención de la enfermedad coronaria en la práctica clínica ${ }^{27,28}$.

Con estas tablas podemos realizar una estimación del riesgo coronario, definido como IAM no fatal y muerte coronaria; utiliza las siguientes variables: edad (35-74 años), sexo, tabaco (sí/no), presión arterial sistólica, cociente colesterol total/ HDL-colesterol y diabetes (sí/no) (existen 2 tablas diferentes respecto a si el paciente es o no diabético).

El manejo es similar al de las tablas europeas, obteniendo el riesgo en un gráfico dividido por 3 líneas que equivalen al 15,20 y $30 \%$ respectivamente; el cociente colesterol total/HDL-colesterol se calcula directamente en la tabla a través de un normograma (Fig. 7).

No son apropiadas para el cálculo de riesgo cardiovascular si presenta: enfermedad coronaria $u$ otra enfermedad arterioesclerótica, hipercolesterolemia familiar, HTA establecida (PAS $>160 \mathrm{mmHg}$ o PAD >100 mmHg) con lesiones de órgano diana, insuficiencia renal o diabetes con lesiones órgano diana; el riesgo es mayor que el reflejado en las 
Probabilidad de evento coronario en 2 años en hombres de 35-74 años sin enfermedad cardiovascular previa.

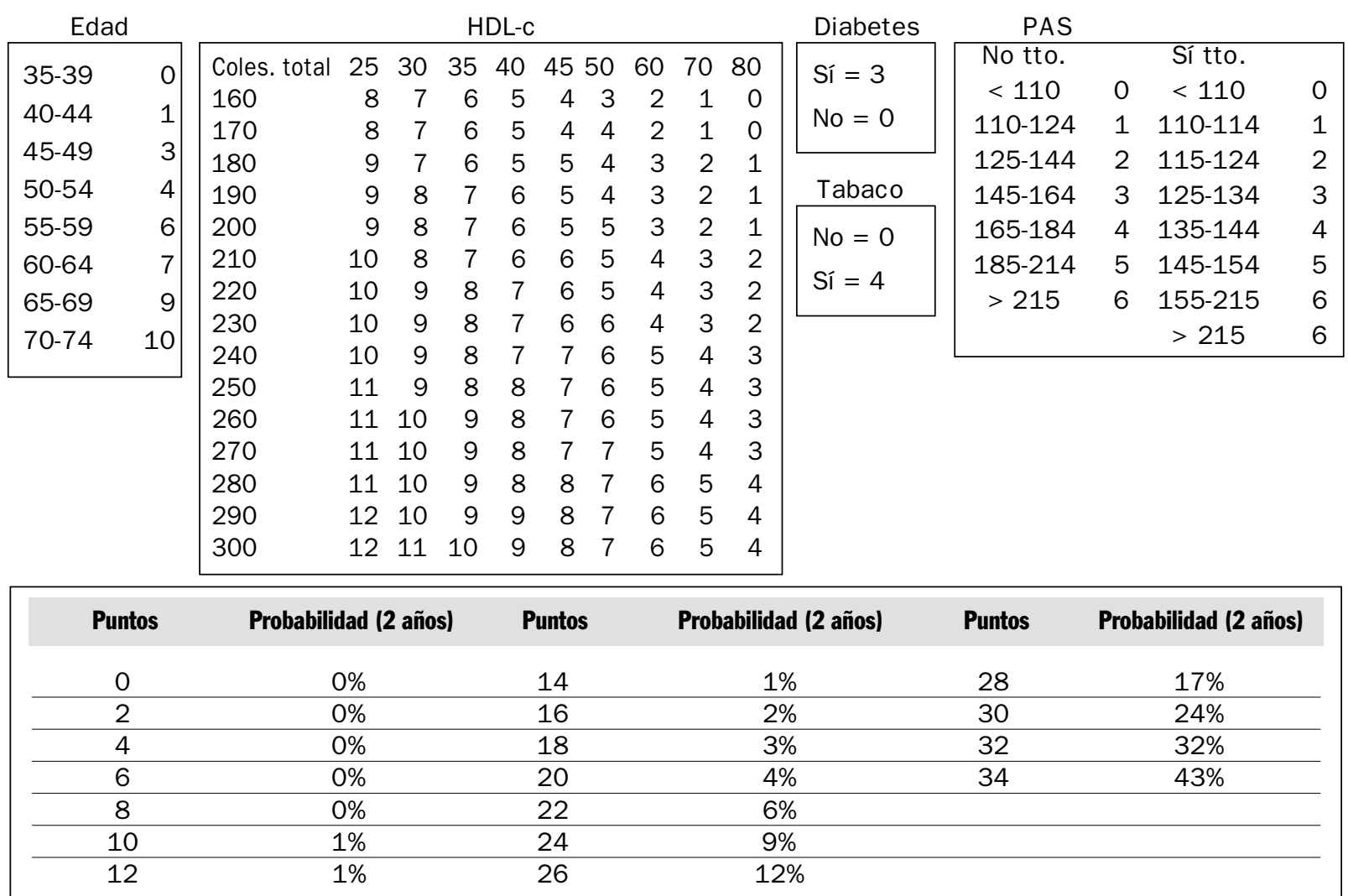

Probabilidad de recidiva coronaria en los 2 años en hombres de 45-74 años con enfermedad coronaria o trombosis cerebral

\begin{tabular}{|c|c|c|c|c|c|c|c|c|c|c|c|c|}
\hline \multicolumn{2}{|c|}{ Edad } & \multicolumn{10}{|c|}{ HDL-C } & \multirow{4}{*}{$\begin{array}{l}\text { Diabetes } \\
\text { Sí }=4 \\
\text { No }=0\end{array}$} \\
\hline $35-39$ & 0 & Coles. total & 25 & 30 & 35 & 40 & 45 & 50 & 60 & 70 & 80 & \\
\hline $40-44$ & 1 & 160 & 10 & 9 & 7 & 6 & 5 & 4 & 3 & 1 & 0 & \\
\hline 40-44 & 1 & 170 & 11 & 9 & 8 & 7 & 6 & 5 & 3 & 2 & 1 & \\
\hline $45-49$ & 3 & 180 & 11 & 10 & 8 & 7 & 6 & 5 & 4 & 2 & 1 & \\
\hline $50-54$ & 4 & 190 & 12 & 10 & 9 & 8 & 7 & 6 & 5 & 3 & 2 & \\
\hline $55-59$ & 6 & 200 & 12 & 11 & 9 & 8 & 7 & 6 & 5 & 3 & 2 & \\
\hline $60-64$ & 7 & 210 & 13 & 11 & 10 & 9 & 7 & 7 & 5 & 4 & 2 & \\
\hline $65-60$ & a & 220 & 13 & 11 & 10 & 9 & 8 & 7 & 5 & 4 & 3 & \\
\hline $65-65$ & 9 & 230 & 13 & 12 & 10 & 9 & 8 & 7 & 6 & 4 & 3 & \\
\hline $70-74$ & 10 & 240 & 14 & 12 & 11 & 10 & 9 & 8 & 6 & 5 & 4 & \\
\hline & & 250 & 14 & 13 & 11 & 10 & 9 & 8 & 6 & 5 & 4 & \\
\hline & & 260 & 15 & 13 & 12 & 10 & 9 & 8 & 7 & 5 & 4 & \\
\hline & & 270 & 15 & 13 & 12 & 11 & 10 & 9 & 7 & 6 & 5 & \\
\hline & & 280 & 15 & 14 & 12 & 11 & 10 & 9 & 7 & 6 & 5 & \\
\hline & & 290 & 16 & 14 & 13 & 11 & 10 & 9 & 8 & 6 & 5 & \\
\hline & & 300 & 16 & 14 & 13 & 12 & 11 & 10 & 8 & 7 & 6 & \\
\hline
\end{tabular}

\begin{tabular}{|cccccc|}
\hline Puntos & Probabilidad (2 años) & Puntos & Probabilidad (2 años) & Puntos & Probabilidad (2 años) \\
\hline 0 & $3 \%$ & 10 & $7 \%$ & 20 & $14 \%$ \\
\hline 2 & $4 \%$ & 12 & $8 \%$ & 22 & $17 \%$ \\
\hline 4 & $4 \%$ & 14 & $9 \%$ & 24 & $19 \%$ \\
\hline 6 & $5 \%$ & 16 & $11 \%$ & 26 & $22 \%$ \\
\hline 8 & 18 & $13 \%$ & 28 & $25 \%$ \\
\hline
\end{tabular}

(Fuente: cita bibliográfica 22)

Figura 4 Nuevas tablas de Framingham (D’Agostino) en hombres; arriba, prevención primaria; abajo, prevención secundaria. 


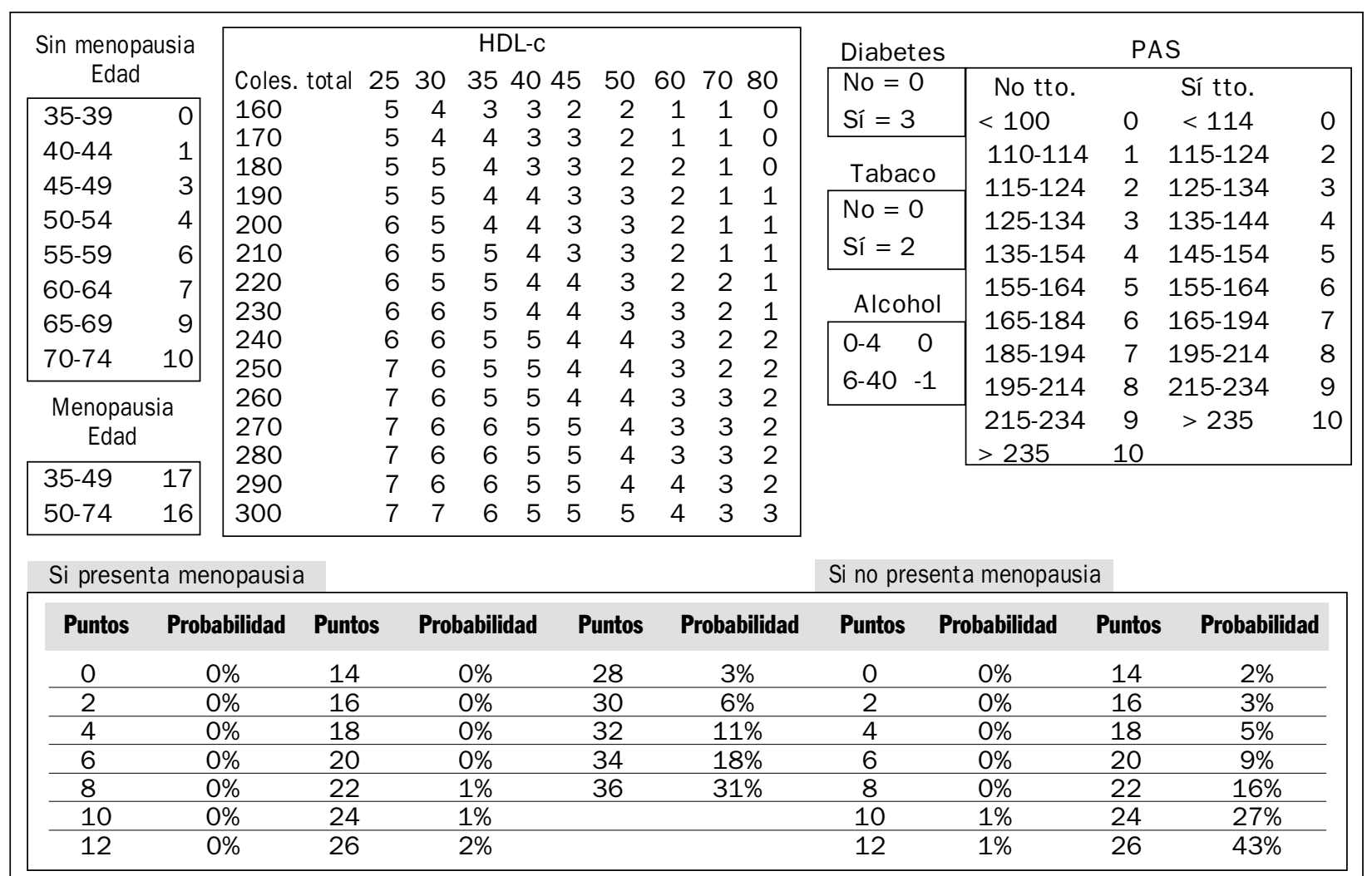

Probabilidad de enfermedad coronaria a los 2 años en mujeres entre 35-74 años sin enfermedad cardiovascular.

Modelo sin triglicéridos.

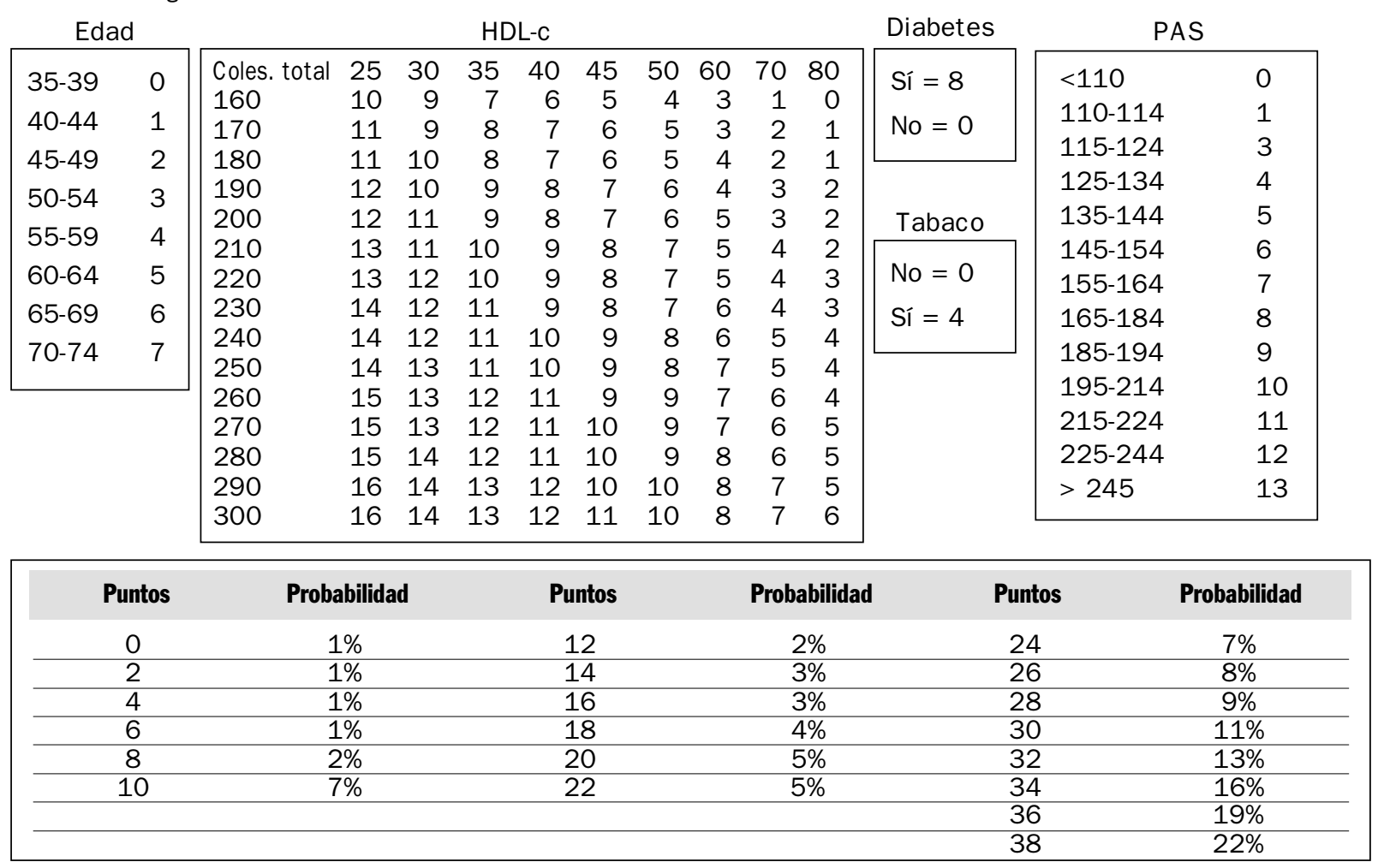

Probabilidad de recidiva enfermedad coronaria a los 2 años en mujeres entre 35-74 años con enfermedad coronaria previa 0 trombosis cerebral.

(Fuente: cita bibliográfica 22)

Figura 5 Nuevas tablas de riesgo de Framingham (D’Agostino) en mujeres; arriba, prevención primaria; abajo, prevención secundaria. 

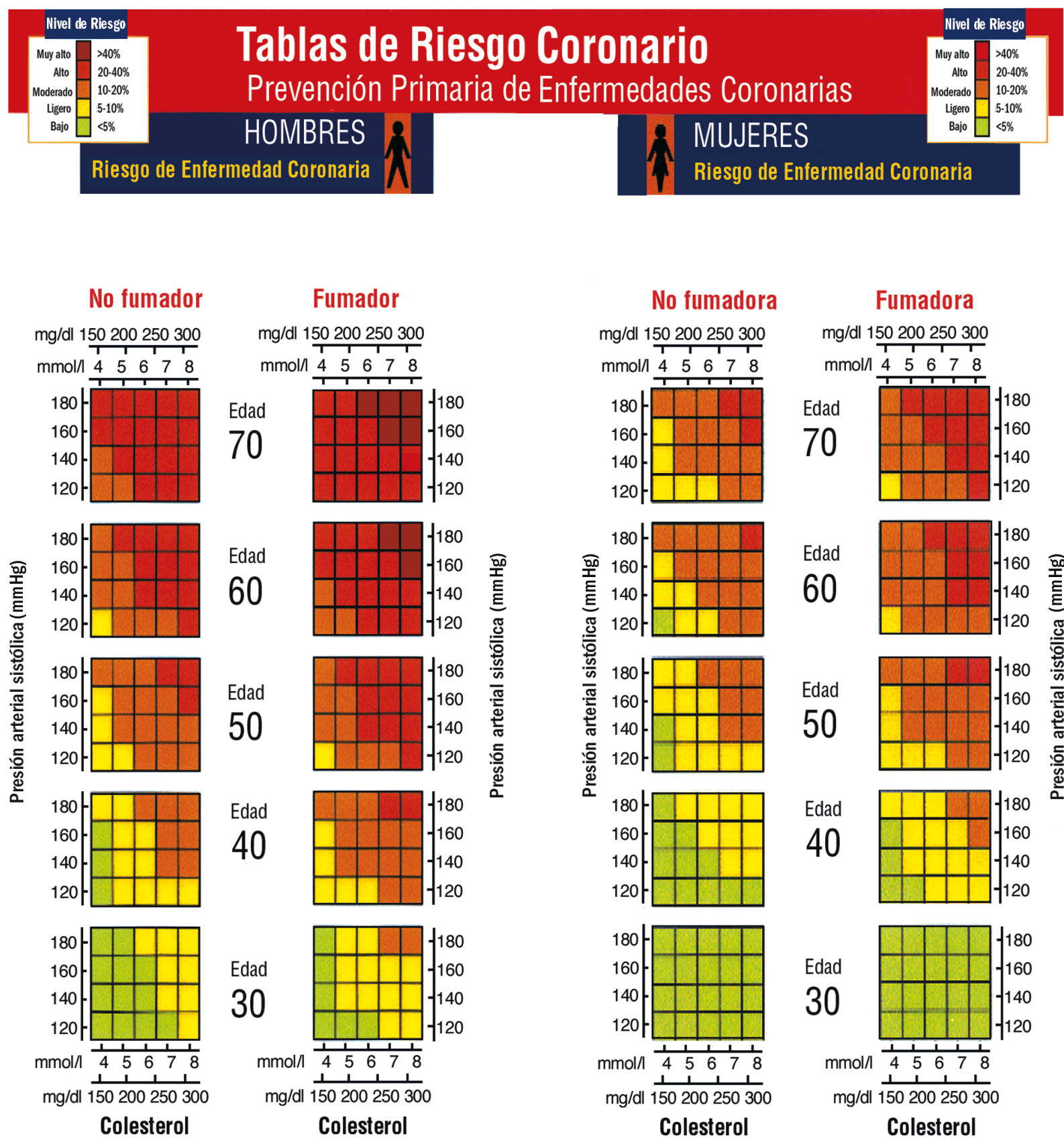

(Fuente: cita bibliográfica 2)

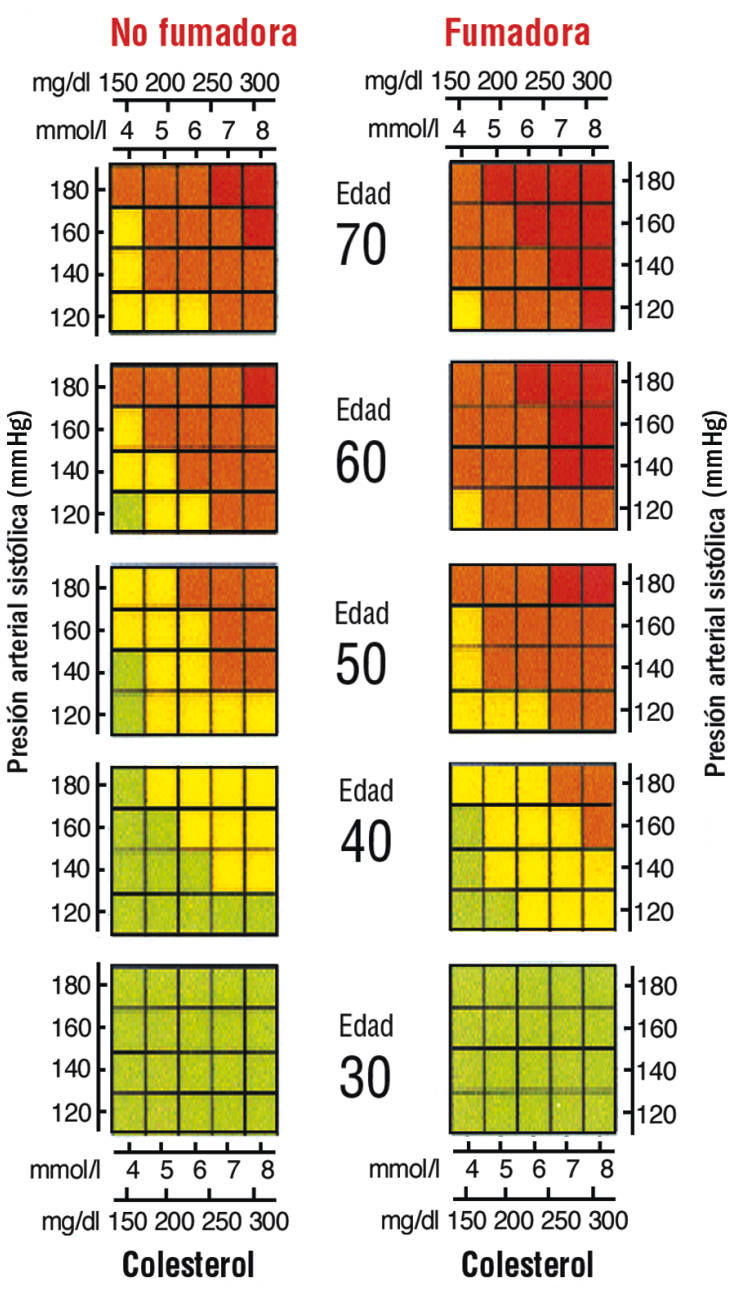

Figura 6

Tablas de riesgo de las Sociedades Europeas.

tablas, si: historia familiar de enfermedad coronaria precoz $(<55$ años en hombres y de 65 años en mujeres), triglicéridos altos, intolerancia a la glucosa, mujeres con menopausia precoz o si la edad del paciente se aproxima a la siguiente categoría.

Se considera alto riesgo si la probabilidad de presentar un evento a los 10 años es superior al
$15 \%$ (equivalente a un $20 \%$ de riesgo cardiovascular); de todas formas se considera prioritario intervenir en aquéllos de un riesgo superior al 30\% y si hay recursos económicos seguir con los de riesgo $>15 \%$.

La diferencia fundamental con las tablas de las sociedades europeas es la inclusión, no solamente 


\section{Joint British Societies \\ coronary risk prediction chart}

\section{Diabetes}
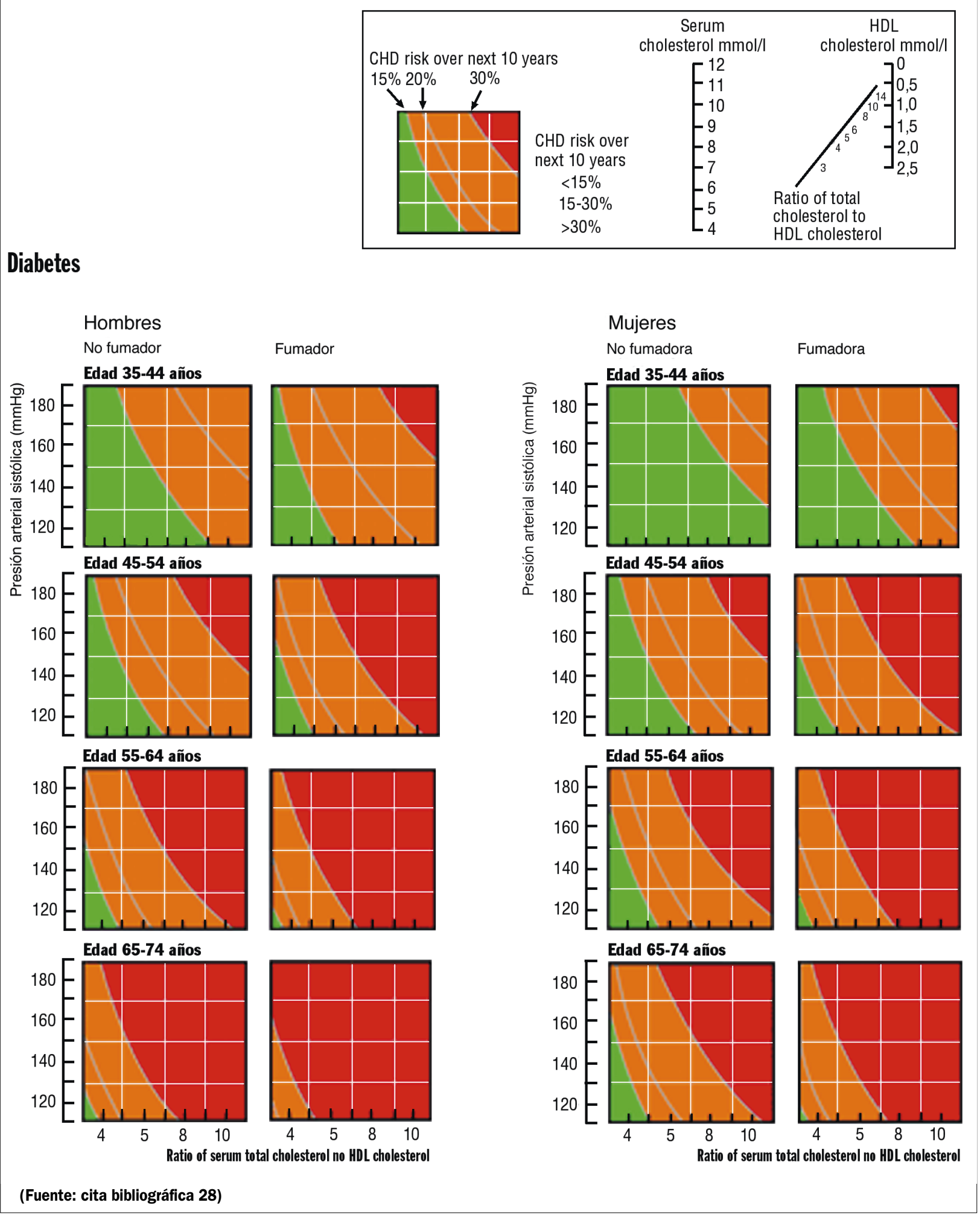

(Fuente: cita bibliográfica 28)

Figura 7

Tablas de riesgo de las Sociedades Británicas. 
del HDL-colesterol, sino del cociente colesterol total/HDL-colesterol, que según diferentes estudios es un mejor predictor de enfermedad coronaria que el colesterol total solo o el LDL-colesterol ${ }^{16,29}$.

\section{TABLAS DE RIESGO DE NUEVA ZELANDA}

Estas tablas no son una guía para el manejo del riesgo cardiovascular en general, sino que se utilizan para ver el beneficio del tratamiento con fármacos de la HTA y el colesterol, dos de los factores de riesgo más importantes, junto con el tabaco; expresan el riesgo cardiovascular definido como muerte coronaria, IAM, angina, accidente isquémico tran sitorio, accidente cerebrovascular fatal o no fatal, insuficiencia cardiaca y enfermedad vascular peri férica, en un periodo de 5 años ${ }^{30}$.

Las variables que utiliza son la edad (40-70 años), sexo, diabetes (sí/no), tabaco (sí/no), cociente colesterol total/ HDL-colesterol, presión arterial sistólica y diastólica (si caen en diferentes categorías se debe aplicar la mayor) (Fig. 8) ${ }^{31}$.

En caso de que el colesterol sea mayor de 8-9 $\mathrm{mmol} / \mathrm{l}$ o el cociente de colesterol total/ HDL-colesterol mayor de 8-9 o la tensión arterial sea mayor de 170-180/100-105 mmHg, las tablas de riesgo infraestiman el verdadero riesgo de ese individuo.

El verdadero interés de estas tablas es que podemos ver reflejado el beneficio del tratamiento en base a eventos cardiovasculares que se previenen por cada 100 tratados en 5 años y el número de pacientes a tratar también durante 5 años para evitar un evento (NNT), asumiendo un descenso del colesterol con tratamiento del $20 \%$ y un descenso de la presión arterial sistólica de 10-15 $\mathrm{mmHg}$ o de presión arterial diastólica de 5-8 $\mathrm{mmHg}^{31}$.

No se deben aplicar en pacientes con enfermedad cardiovascular manifiesta o con HVI, diagnosticada por ECG, ya que se asume que estos casos presentan un riesgo superior al $20 \%$ a los 5 años; tampoco se debe aplicar si existe hiperlipemia genética familiar o diabéticos con microalbuminuria (MAU); aquellos pacientes con historia familiar de enfermedad coronaria prematura $(<55$ años en varones y de 65 años en mujeres) o con obesidad, van a tener un riesgo más alto que el indicado en las tablas.

El cálculo de riesgo cardiovascular a 5 años en pacientes con hipertensión arterial es otra de las ventajas de estas tablas, ya que se ajusta mejor a los resultados obtenidos en los distintos ensayos clínicos de HTA, que suelen tener esta duración.

\section{TABLAS DE RIESGO DE SHEFFIELD}

El objetivo de estas tablas es detectar qué pacientes se pueden beneficiar de un tratamiento con estatinas en base a su cifra de colesterol total y su riesgo coronario; a raíz de la publicación del estudio $4 \mathrm{~S}^{32}$ realizado en prevención secundaria con simvastatina, y extrapolando los datos a la prevención primaria, se podía obtener beneficio si el riesgo de muerte coronaria era mayor de un $1,5 \%$ anual (riesgo de los pacientes tratados con placebo en este ensayo); posteriormente y a raíz del estudio WOSCOPS $^{33}$, se modificaron las tablas, siendo el riesgo mínimo para tratar de un 3\% anual.

Se calculó por lo tanto, usando las funciones de riesgo de Framingham, cuáles eran las concentraciones de colesterol total que provocaban un riesgo de morir de un 1,5\% (en las modificadas, del 3\%), en base a una serie de variables, como: edad, sexo, tabaco (sí/no), HTA, diabetes mellitus (presente/ ausente), HVI y valores medios de HDL-colesterol; el límite de edad se cifraba, al igual que en el $4 \mathrm{~S}$, en 70 años ${ }^{34,35}$.

Recientemente se han publicado unas nuevas tablas de Sheffield ${ }^{36}$, ajustándose a las recomendaciones de las Sociedades Británicas en la prevención de la enfermedad coronaria; utiliza como variables la edad (28-70 años en hombres y 36-70 años en mujeres), sexo, tabaco (sí/no), diabetes (sí/no), HTA, cociente colesterol total: HDL-colesterol, indicando en cada columna un riesgo del 15 o del $30 \%$ a los 10 años (Fig. 9).

La forma de utilizar las tablas es como sigue: se elige la tabla correspondiente a hombre o mujer, y una vez encontrada la columna correcta respecto a HTA, diabetes y tabaco, se va a la fila correspondiente a la edad; si encontramos alguna entrada, el colesterol total y HDL-colesterol debe ser medido para el cálculo del cociente; si éste nos da un riesgo del $15 \%$ se debe considerar el tratamiento de la HTA moderada (sistólica 140-159 o diastólica 9099) y con aspirina (según las recomendaciones de las Sociedades Británicas de Hipertensión, Cardiología, Diabetes y la Asociación Británica de Hiperlipemias) $)^{27}$; si el cociente colesterol total/ HDL-colesterol confiere un riesgo del $30 \%$, se debe considerar un tratamiento con estatinas si el colesterol total es mayor o igual a 5,0 $\mathrm{mmol} / 1$; si no encontramos entrada en la intersección de los factores de riesgo y la edad, no debemos medir los lípidos ya que independientemente de la cifra obtenida, nunca llegaría al umbral de riesgo para ser tratado con fármacos, salvo que se sospeche una hiperlipemia familiar.

Se debe destacar que no se debe utilizar en caso de prevención secundaria, y que las tablas infraestiman el riesgo en caso de: HVI, historia familiar de enfermedad coronaria prematura e hiperlipemia familiar.

Si utilizamos como "patrón oro" la tabla de riesgo de Framingham, las nuevas tablas de Sheffield, tienen una sensibilidad del $97 \%$ y especificidad del 


\section{New Zealand cardiovascular risk prediction charts}

\section{Hombres}
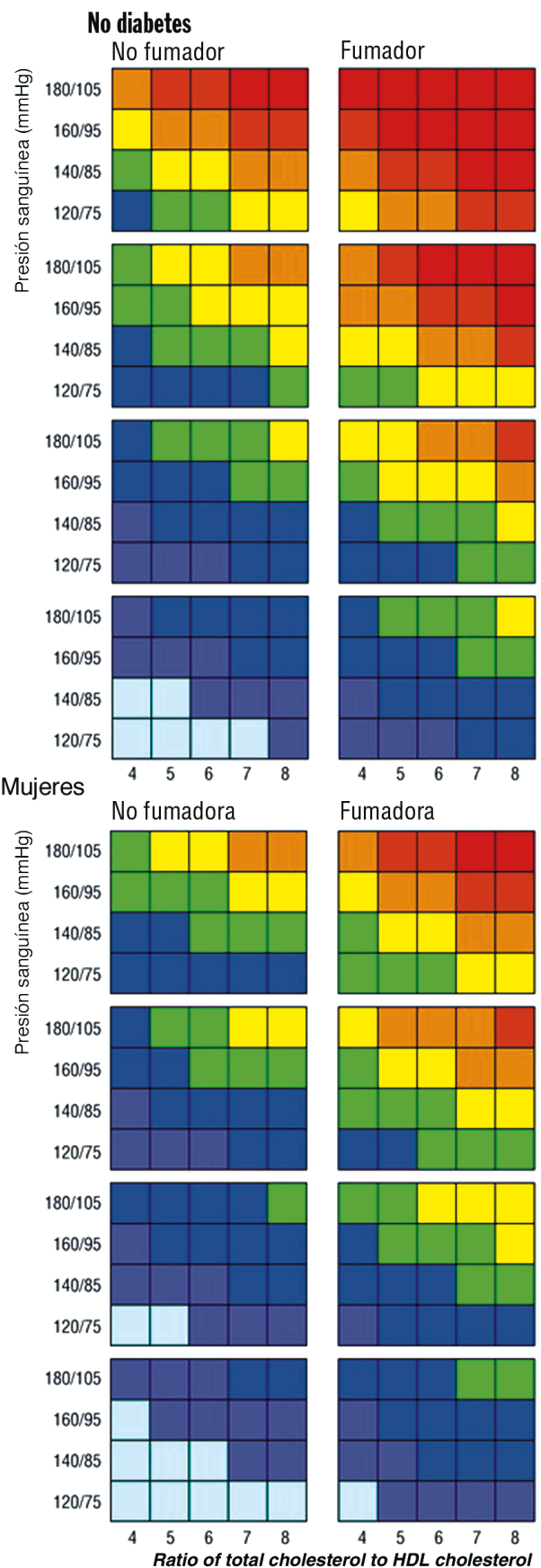

(Fuente: cita bibliográfica 31)
Fumador
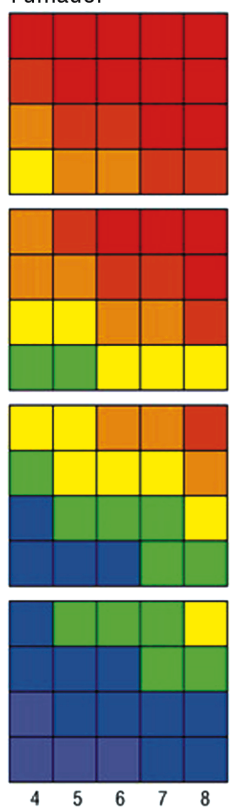

Fumadora
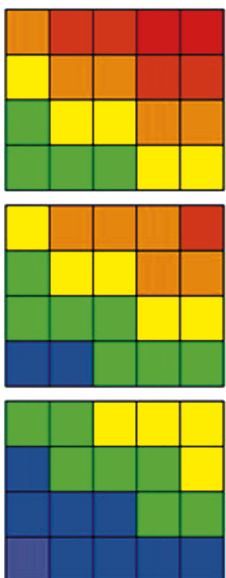

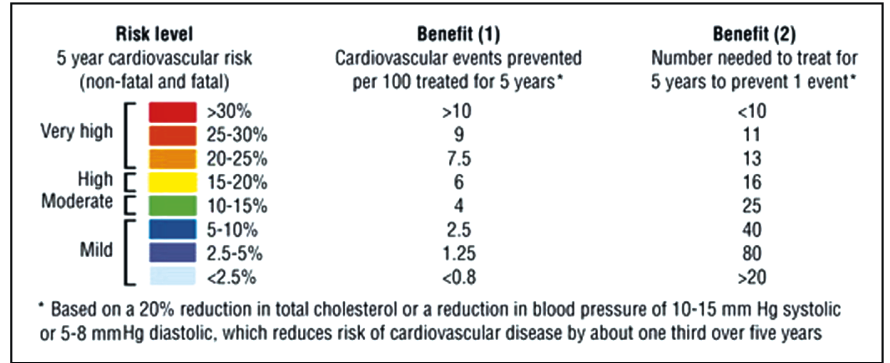

Edad 70

\section{Diabetes}

No fumador

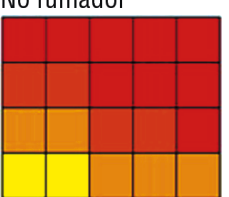

Fumador

Edad 60
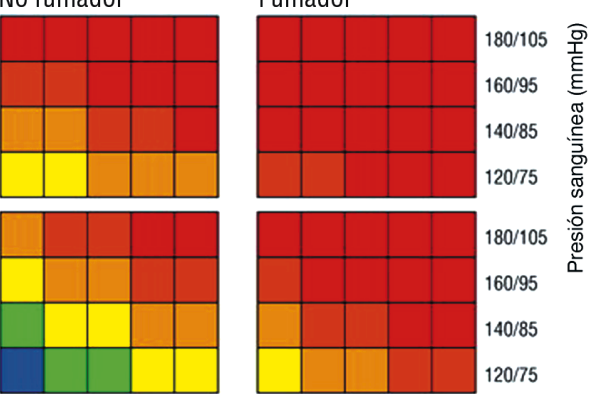

Edad 50
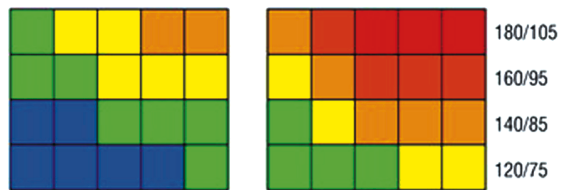

Edad 40
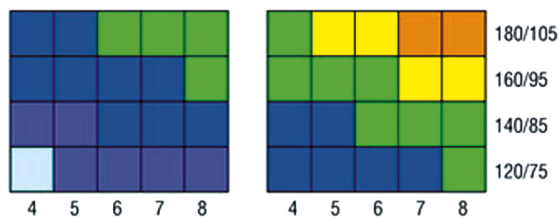

No fumadora

Fumadora

Edad 70
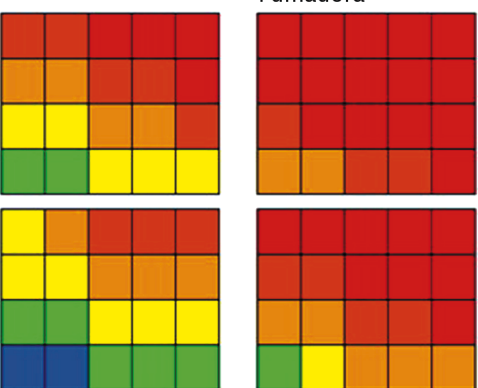

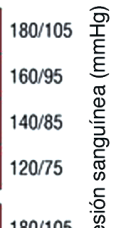

Edad 60
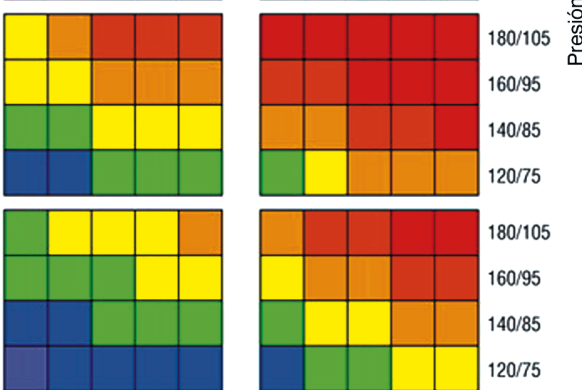

Edad 40

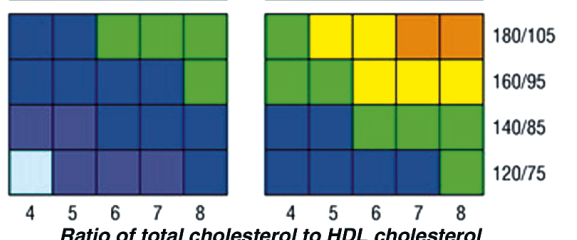

Figura 8

Tablas de riesgo de Nueva Zelanda. 


\begin{tabular}{|c|c|c|c|c|c|c|c|c|c|c|c|c|c|c|c|c|}
\hline \multirow{3}{*}{$\begin{array}{l}\text { Men Tot } \\
\text { Hypertension } \\
\text { Smoking } \\
\text { Diabetes } \\
\text { CHD risk } \\
\end{array}$} & I: HD & choles & erol ra & & & & & & & & & & & & & \\
\hline & \multicolumn{2}{|c|}{$\begin{array}{l}\text { Yes } \\
\text { Yes } \\
\text { Yes } \\
\end{array}$} & \multicolumn{2}{|c|}{$\begin{array}{l}\text { No } \\
\text { Yes } \\
\text { Yes } \\
\end{array}$} & \multicolumn{2}{|c|}{$\begin{array}{l}\text { Yes } \\
\text { Yes } \\
\text { No }\end{array}$} & \multicolumn{2}{|c|}{$\begin{array}{l}\text { Yes } \\
\text { No } \\
\text { Yes }\end{array}$} & \multicolumn{2}{|c|}{$\begin{array}{l}\text { No } \\
\text { Yes } \\
\text { No }\end{array}$} & \multicolumn{2}{|c|}{$\begin{array}{l}\text { No } \\
\text { No } \\
\text { Yes }\end{array}$} & \multicolumn{2}{|c|}{$\begin{array}{l}\text { Yes } \\
\text { No } \\
\text { No }\end{array}$} & \multicolumn{2}{|c|}{$\begin{array}{l}\text { No } \\
\text { No } \\
\text { No }\end{array}$} \\
\hline & $15 \%$ & $30 \%$ & $15 \%$ & $30 \%$ & $15 \%$ & $30 \%$ & $15 \%$ & $30 \%$ & $15 \%$ & $30 \%$ & $15 \%$ & $30 \%$ & $15 \%$ & $30 \%$ & $15 \%$ & $30 \%$ \\
\hline Age & 2.0 & 3.0 & 2.0 & 3.6 & 2.1 & 3.8 & 2.4 & 4.4 & 2.5 & 4.6 & 2.9 & 5.3 & 3.1 & 5.6 & 3.7 & 6.7 \\
\hline 68 & 2.0 & 3.2 & 2.1 & 3.8 & 2.2 & 4.1 & 2.6 & 4.7 & 2.7 & 4.8 & 3.0 & 5.6 & 3.3 & 6.0 & 3.9 & 7.1 \\
\hline 66 & 2.0 & 3.4 & 2.2 & 4.0 & 2.4 & 4.3 & 2.7 & 5.0 & 2.8 & 5.2 & 3.2 & 5.9 & 3.5 & 6.3 & 4.1 & 7.6 \\
\hline 64 & 2.0 & 3.6 & 2.4 & 4.3 & 2.5 & 4.6 & 2.9 & 5.3 & 3.0 & 5.5 & 3.5 & 6.3 & 3.7 & 6.8 & 4.4 & 8.1 \\
\hline 62 & 2.1 & 3.8 & 2.5 & 4.6 & 2.7 & 4.9 & 3.1 & 5.6 & 3.2 & 5.9 & 3.7 & 6.7 & 3.9 & 7.2 & 4.7 & 8.6 \\
\hline 60 & 2.2 & 4.1 & 2.7 & 4.9 & 2.9 & 5.2 & 3.3 & 6.0 & 3.4 & 6.3 & 3.9 & 7.2 & 4.2 & 7.7 & 5.0 & 9.2 \\
\hline 58 & 2.4 & 4.4 & 2.9 & 5.3 & 3.1 & 5.6 & 3.5 & 6.5 & 3.7 & 6.7 & 4.2 & 7.7 & 4.5 & 8.3 & 5.4 & 9.9 \\
\hline 56 & 2.6 & 4.7 & 3.1 & 5.7 & 3.3 & 6.0 & 3.8 & 7.0 & 4.0 & 7.2 & 4.6 & 8.3 & 4.9 & 8.9 & 5.8 & 10.6 \\
\hline 54 & 2.8 & 5.1 & 3.3 & 6.1 & 3.6 & 6.5 & 4.1 & 7.5 & 4.3 & 7.8 & 4.9 & 9.0 & 5.2 & 9.6 & 6.3 & - \\
\hline 52 & 3.0 & 5.5 & 3.6 & 6.6 & 3.9 & 7.0 & 4.4 & 8.1 & 4.6 & 8.4 & 5.3 & 9.7 & 5.7 & 10.4 & 6.8 & - \\
\hline 50 & 3.3 & 6.0 & 3.9 & 7.1 & 4.2 & 7.6 & 4.8 & 8.8 & 5.0 & 9.1 & 5.7 & 10.5 & 6.1 & - & 7.3 & - \\
\hline 48 & 3.6 & 6.5 & 4.3 & 7.8 & 4.5 & 8.3 & 5.2 & 9.6 & 5.4 & 9.9 & 6.3 & - & 6.7 & - & 8.0 & - \\
\hline 46 & 3.9 & 7.1 & 4.6 & 8.5 & 5.0 & 9.1 & 5.7 & 10.4 & 5.9 & 10.8 & 6.8 & - & 7.3 & - & 8.7 & - \\
\hline 44 & 4.3 & 7.8 & 5.1 & 9.3 & 5.4 & 9.9 & 6.3 & $\cdot$ & 6.5 & $\cdot$ & 7.5 & - & 8.0 & - & 9.6 & - \\
\hline 42 & 4.7 & 8.6 & 5.6 & 10.2 & 6.0 & 10.9 & 6.9 & - & 7.2 & $\cdot$ & 8.2 & - & 8.8 & - & 10.5 & - \\
\hline 40 & 5.2 & 9.5 & 6.2 & - & 6.6 & - & 7.6 & - & 7.9 & - & 9.1 & - & 9.7 & - & & \\
\hline 38 & 5.8 & 10.5 & 6.9 & - & 7.3 & - & 8.5 & - & 8.8 & - & 10.1 & - & 10.8 & - & & \\
\hline 36 & 6.4 & $\cdot$ & 7.7 & - & 8.2 & - & 9.5 & - & 9.8 & - & & & & & & \\
\hline 34 & 7.2 & - & 8.6 & - & 9.2 & - & 10.6 & . & & & & & & & & \\
\hline 32 & 8.2 & - & 9.8 & - & 10.5 & - & & & & & & & & & & \\
\hline $\begin{array}{l}30 \\
28 \\
\end{array}$ & $\begin{array}{c}9.4 \\
10.8\end{array}$ & - & & & & & & & & & & & & & & \\
\hline
\end{tabular}

\begin{tabular}{|c|c|c|c|c|c|c|c|c|c|c|c|c|c|c|c|c|c|}
\hline & & & & & & & & & & & & \multicolumn{6}{|c|}{ Total: HDL cholesterol ratio } \\
\hline \multirow{2}{*}{\multicolumn{2}{|c|}{$\begin{array}{l}\text { Women To } \\
\text { Hypertension } \\
\text { Smoking } \\
\text { Diabetes } \\
\text { CHD risk }\end{array}$}} & \multicolumn{2}{|c|}{$\begin{array}{l}\text { Yes } \\
\text { Yes } \\
\text { Yes }\end{array}$} & \multicolumn{2}{|c|}{$\begin{array}{l}\text { No } \\
\text { Yes } \\
\text { Yes }\end{array}$} & \multicolumn{2}{|c|}{$\begin{array}{l}\text { Yes } \\
\text { No } \\
\text { Yes }\end{array}$} & \multicolumn{2}{|c|}{$\begin{array}{l}\text { Yes } \\
\text { Yes } \\
\text { No }\end{array}$} & \multicolumn{2}{|c|}{$\begin{array}{l}\text { No } \\
\text { No } \\
\text { Yes }\end{array}$} & \multicolumn{2}{|c|}{$\begin{array}{l}\text { No } \\
\text { Yes } \\
\text { No }\end{array}$} & \multicolumn{2}{|c|}{$\begin{array}{l}\text { Yes } \\
\text { No } \\
\text { No }\end{array}$} & \multicolumn{2}{|c|}{$\begin{array}{l}\text { No } \\
\text { No } \\
\text { No }\end{array}$} \\
\hline & & $15 \%$ & $30 \%$ & $15 \%$ & $30 \%$ & $15 \%$ & $30 \%$ & $15 \%$ & $30 \%$ & $15 \%$ & $30 \%$ & $15 \%$ & $30 \%$ & $15 \%$ & $30 \%$ & $15 \%$ & $30 \%$ \\
\hline \multirow[t]{18}{*}{ Age } & 70 & 2.3 & 4.1 & 2.7 & 4.9 & 3.3 & 6.1 & 3.8 & 7.0 & 4.0 & 7.2 & 4.6 & 8.3 & 5.6 & 10.2 & 6.7 & - \\
\hline & 68 & 2.3 & 4.2 & 2.7 & 5.0 & 3.4 & 6.1 & 3.9 & 7.0 & 4.0 & 7.3 & 4.6 & 8.4 & 5.7 & - & 6.8 & - \\
\hline & 66 & 2.3 & 4.2 & 2.8 & 5.1 & 3.4 & 6.2 & 3.9 & 7.1 & 4.1 & 7.4 & 4.7 & 8.5 & 5.7 & - & 6.9 & - \\
\hline & 64 & 2.4 & 4.3 & 2.8 & 5.2 & 3.5 & 6.4 & 4.0 & 7.3 & 4.2 & 7.6 & 4.8 & 8.7 & 5.9 & - & 7.0 & - \\
\hline & 62 & 2.4 & 4.4 & 2.9 & 5.3 & 3.6 & 6.5 & 4.1 & 7.5 & 4.3 & 7.8 & 4.9 & 9.0 & 6.0 & - & 7.2 & - \\
\hline & 60 & 2.5 & 4.6 & 3.0 & 5.5 & 3.7 & 6.7 & 4.2 & 7.7 & 4.4 & 8.1 & 5.1 & 9.3 & 6.2 & - & 7.4 & - \\
\hline & 58 & 2.6 & 4.8 & 3.1 & 5.7 & 3.8 & 7.0 & 4.4 & 8.0 & 4.6 & 8.4 & 5.3 & 9.6 & 6.5 & - & 7.8 & - \\
\hline & 56 & 2.7 & 5.0 & 3.3 & 6.0 & 4.0 & 7.4 & 4.6 & 8.4 & 4.8 & 8.8 & 5.5 & 10.1 & 6.8 & - & 8.1 & - \\
\hline & 54 & 2.9 & 5.3 & 3.5 & 6.3 & 4.3 & 7.8 & 4.9 & 8.9 & 5.1 & 9.3 & 5.8 & - & 7.2 & - & 8.6 & - \\
\hline & 52 & 3.1 & 5.6 & 3.7 & 6.8 & 4.5 & 8.3 & 5.2 & 9.5 & 5.4 & 9.9 & 6.2 & - & 7.7 & - & 9.2 & - \\
\hline & 50 & 3.3 & 6.1 & 4.0 & 7.3 & 4.9 & 9.0 & 5.6 & - & 5.9 & - & 6.7 & - & 8.3 & - & 9.9 & - \\
\hline & 48 & 3.6 & 6.6 & 4.3 & 7.9 & 5.3 & 9.8 & 6.1 & - & 6.4 & - & 7.3 & - & 9.0 & - & & \\
\hline & 46 & 4.0 & 7.3 & 4.8 & 8.8 & 5.9 & - & 6.8 & . & 7.1 & . & 8.1 & . & 10.0 & - & & \\
\hline & 44 & 4.5 & 8.2 & 5.4 & 9.8 & 6.6 & - & 7.6 & - & 7.9 & - & 9.1 & - & & & & \\
\hline & 42 & 5.1 & 9.4 & 6.1 & - & 7.5 & - & 8.6 & - & 9.0 & - & 10.3 & - & & & & \\
\hline & 40 & 5.9 & - & 7.1 & - & 8.7 & - & 10.0 & - & & & & & & & & \\
\hline & 38 & 7.0 & - & 8.4 & - & & & & & & & & & & & & \\
\hline & 36 & 8.5 & - & 10.2 & - & & & & & & & & & & & & \\
\hline
\end{tabular}

(Fuente: cita bibliográfica 36)

Figura 9 Nuevas tablas de riesgo de Sheffield.

95\% para un riesgo coronario mayor o igual al 15\%; si consideramos el umbral en mayor o igual al 30\%, la sensibilidad es del 82\% y especificidad del 99\%.

La ventaja de estas tablas es que se pueden utilizar como herramienta de "screening" para los lípi- dos, ya que en población de 35-44 años un 39\% de hombres y $89 \%$ de mujeres, no necesitarán conocer su cifra de lípidos, ya que nunca llegarían al porcentaje de riesgo requerido para el tratamiento farmacológico; así y todo, hoy en día se debe replan- 
tear esta cuestión ante las evidencias del beneficio del tratamiento con estatinas.

Entre los inconvenientes se puede resaltar que es el método más estricto ${ }^{37}$ a la hora de tratar el colesterol con estatinas (riesgo del 3\% anual, en principio), comparado con las tablas Europeas (riesgo del $2 \%$ anual) o el NCEP, lo cual está basado en el coste- efectividad del tratamiento con fármacos hipolipemiantes ${ }^{38}$; además, según algunos estudios, no es la más fácil de aplicar por los médicos generales o enfermeras, prefiriendo por ejemplo las tablas de riesgo de Nueva Zelanda o las británicas ${ }^{39}$.

\section{DISCUSIÓN}

A lo largo de este capítulo hemos visto diferentes tablas para el cálculo del riesgo cardiovascular global de nuestros pacientes, etapa fundamental a la hora de intentar reducir el riesgo en aquellos casos en que éste sea alto ${ }^{40}$; el primer problema que nos vamos a encontrar es elegir la tabla de riesgo más adecuada a nuestro medio; debería de cumplir, por lo menos, las siguientes características: fácil de utilizar y emplear factores de riesgo contrastados en nuestra población; en principio, creemos que son más adecuadas las tablas representadas en gráficos de colores según riesgo, ya que el paciente comprenderá mejor el beneficio del tratamiento de sus factores de riesgo; es importante también las variables que se utilizan, ya que por un lado, cuantos más factores de riesgo más complicada será la tabla, y por otro pueden existir factores de riesgo con un peso específico importante, como por ejemplo el HDL-colesterol en España.

Las tablas de Framingham originales y por Categorías, dado que son obtenidas de una población con un riesgo de enfermedad cardiovascular mucho más alto que la española, podrían supraestimar el riesgo en nuestra población; de ahí que quizás sería conveniente en todo caso, utilizar la puntuación de riesgo de "eventos duros" del Framingham por categorías (Grundy); las nuevas tablas de Framingham (D'Agostino), sobre todo en mujeres, creemos que utilizan muchos factores de riesgo, lo que complica un poco su aplicación en la práctica clínica; en su favor, está el ser las únicas con aplicación en prevención secundaria.

Las tablas de las Sociedades Europeas son las más fáciles de utilizar y además su representación en gráficos de color según riesgo individual, les confiere un atractivo para su entendimiento por parte del paciente, así como de ayuda a la hora de corregir sus factores de riesgo; sin embargo, su aplicación en España, a pesar de ser la recomendada por el Consenso de Colesterolemia 2000, crea cierta incertidumbre, ya que no tiene en cuenta el HDL-colesterol.

Las tablas de las Sociedades Británicas son parecidas a las Europeas en lo que a representación gráfica se refiere, pero tienen en cuenta no solamente el HDL-colesterol, sino el cociente colesterol total/HDL-colesterol, mejor predictor de enfermedad coronaria; en la práctica clínica, esto no es problema ya que con los datos de las 2 variables y a través de un normograma se puede calcular fácilmente dicho cociente.

Las tablas de riesgo de Nueva Zelanda y de Sheffield se basan en el tratamiento de dos factores de riesgo fundamentales como la HTA y el colesterol; la primera tiene como fundamental ventaja el conocer el número de eventos que se previenen si tratamos a 100 personas durante 5 años, así como el número de pacientes a tratar para evitar un evento (NNT); la segunda, es más difícil de aplicar en la práctica clínica, basándose más en un sistema de coste-efectividad del tratamiento con fármacos que en medicina de la evidencia.

Está claro que con sus ventajas e inconvenientes, es mejor utilizar cualquiera de las tablas de riesgo que ninguna y que a pesar de su divulgación, su aplicación por parte del personal sanitario es escasa y que los resultados son muy pobres ${ }^{41}$.

Además las tablas utilizan distintas variables y éste es un proceso muy dinámico, ya que a medida que se analizan nuevos resultados, tendremos que ir incorporando otros factores de riesgo, con el objetivo de acercarnos más a la realidad multifactorial de la arterioesclerosis.

Por último está claro que en poblaciones de menor riesgo coronario, debemos realizar estudios que adapten las predicciones del riesgo cardiovascular, basadas casi todas en resultados obtenidos en anglosajones, a nuestra población ${ }^{42}$.

CORRESPONDENCIA:

A. Álvarez Cosmea

C/ Rafael Altamira, 5, $11^{\circ} \mathrm{A}$

33006 Oviedo 


\section{Bibliografía}

1. Villar Álvarez F, Banegas Banegas JR, Rodríguez Artalejo F, Rey Calero J. Mortalidad Cardiovascular en España y sus comunidades autónomas (1975-1992). Med Clin (Barc) 1998; 110: 321-7.

2. Wood D, De Backer G, Faergeman O, Graham I, Mancia G, Pyörala K. Task Force Report. Prevention of coronary heart disease in clinical practice: Recomendations of the second joint task force of the joint European Societies on coronary prevention. Eur Heart J 1998; 19: 1434-503.

3. Jackson R. Guidelines on preventing cardiovascular disease in clinical practice. BMJ 2000; 320: 659-61.

4. Morey SS. AHA and ACC Outline Approaches to Coronary Disease Risk Assessment. Practice Guidelines. American Family Phisician, 2000; 61: 2534-42.

5. Anderson KM, Wilson PWF, Odell PM, Kannel WB. An Updated coronary risk profile. A Statement for health profesionals. Circulation 1991; 83: 356-62.

6. Grupo de Prevención Cardiovascular del Programa de Actividades Preventivas y Promoción de la Salud (PAPPS) de la Sociedad Española de Medicina Familia y Comunitaria (semFYC). Guía de Prevención Cardiovascular. Madrid: Sersa, 1996

7. Haq IU, Ramsay LE, Yeo WW, Jackson PR, Wallis EJ. Is the Framingham Risk Funtion valid for Norther European populations? A comparasion of methods for estimating absolute coronary risk in high risk men. Heart 1999; 81: 40-6.

8. Robson J, Boomla K, Hart B, Feder G. Estimating cardiovascular risk for primary prevention: outstanding questions for primary care. BMJ, 2000; 320: 702-4.

9. Menotti A, Pudd PE, Lanti M. Comparison of the Framingham risk function- based coronary chart with risk function from an Italian population study. European Heart Journal, 2000; 21: 365- 70.

10. Elosua R, Belmonte E, Sentí M, Masiá R, Marrugat J. ¿Debe diseñarse una estrategia específica de prevención primaria de la cardiopatía isquémica para los países mediterráneos de Europa? Clin Invest Arteriosclerosis 2000; 12: 43-55.

11. Grundy Scott M, Balady Gary J, Criqui Michael H, Fletcher G, Greenland P, Hiratzka Loren F, et al. Primary Prevention of Coronary Heart Disease: Guidance From Framingham. Circulation 1998; 97: 1876-87.

12. Pintó Sala X. Dislipemias y riesgo cardiovascular. Bases para la práctica clínica. Barcelona: Ediciones Mayo, 1997.

13. Haffner SM, Lehto S, Ronnemaa T, Pyorala K, Laakso M. Mortality from coronary heart disease in subjects with type 2 diabetes and in nondiabetic subjects without prior myocardial infarction. N Engl J Med 1998; 339: 229-34.

14. Summmary of the second report of the National Cholesterol Education Program (NCEP). Expert Panel on Detection, Evaluation, and Treatment of High Blood Cholesterol in Adults. JAMA 1993; 269: 3015-23.

15. V Informe (JNC) Joint National Committee. The fifth report of the Joint National Committee on detection, evaluation, and treatment of high blood pressure (JNC-V). Arch Intern Med 1993; 153: 154-83.

16. Wilson Peter WF, D'agostino R, Levy D, Belanger A, Silbershatz H, Kannel W. Prediction of Coronary Heart Disease Using Risk Factor categories. Circulation 1998; 97: 1837-47.

17. Grundy S, Pasternak R, Greenland PH, Smith S, Fuster V. Assessment of Cardiovascular Risk by use of Multiple-Risk-Factor Assesment Equations. ACC/AHA Scientific Statement. Circulation 1999; 100: 1481-92.
18. Grundy S. Primary prevention of coronary heart disease: Selection of patiens for aggressive cholesterol management. American Journal of Medecine, 1999; 107: 2S-6S.

19. Mosca L. Absolute risk assessment in the clinical setting. American Journal of Medecine 1999; 107: 7S-9S.

20. Grundy S, Bazaarre T, Cleeman J, D’Agostino R, Hill M, Houston-Miller N, et al. Prevention Conference V: Beyond secondary prevention: identifying the patient for primary prevention: medical office assessment: Writing Group. Circulation 2000; 101: E3-E11.

21. Kannel WB. Riesgo cardiovascular global en ancianos. Cardiovascular Risk Factors 2000; 9: 217-25.

22. D'Agostino R, Russell H, Huse D, Ellison R, Silbershatz $\mathrm{H}$, Wilson $\mathrm{P}$, et al. Primary and subsequent coronary risk appraisal: New Results from the Framingham Study. Am Heart J 2000; 139: 272-81.

23. Velasco JA, Cosin J, Maroto JM, Muñiz J, Casasnovas JA, Plaza I, et al. Guías de práctica clínica de la Sociedad Española de Cardiología en prevención cardiovascular y rehabi litación cardíaca. Rev Esp Cardiol 2000; 53: 1095-120.

24. Plaza Pérez I, Villar Álvarez F, Mata López P, Pérez Jiménez F, Maiques Galán A, Casasnovas Lenguas JA, et al. Control de la colesterolemia en España, 2000. Un instrumento para la prevención cardiovascular. Rev Esp Cardiol 2000; 53: 815-37.

25. Gómez Gerique JA, Gutierrez-Fuentes JA, Montoya T, Porres A, Rueda A, Avellaneda A, et al. Perfil lipídico de la población española: estudio DRECE (Dieta y Riesgo de Enfermedad Cardiovascular en España). Med Clin (Barc) 1999; 113: 730-5.

26. Assman G, Cullen P, Schulte H. The Münster Heart Study (PROCAM). Results of follow-up at 8 years. Eur Heart J 1998; 19 (Suppl A): 2-11.

27. Wood D, Durrington P, Poulter N, Mcinnes G, Rees A, Wray R. Joint British recomendations on prevention of coronary heart disease in clinical practice. Heart 1998; 80 (Suppl 2): 1-29.

28. British Cardiac Society, British Hyperlipidaemia Association, British Hypertension Society, British Diabetic Association. Joint British recommendations on prevention of coronary heart disease in clinical practice: Summary. BMJ 2000; 320: 705-8.

29. Faergeman O. New British recommendations for prevention of coronary heart disease in clinical practice. Heart 1999; 81: 335

30. National Health Committee. Guidelines for the management of mildly raised blood pressure in New Zealand. Wellington: Ministry of Health, 1995.

31. Jackson R. Updated New Zaeland cardiovascular disease risk- benefit prediction guide. BMJ 2000; 320: 709-10.

32. Scandinavian Simvastatin Survival Study Group. Randomised trial of cholesterol lowering in 4.444 patiets with coronary heart disease: the Scandinavian Simvastatin Survival Study (4S). Lancet 1994; 344: 1383-9.

33. Shepherd J, Cobbe S, Ford I, Isles C, Ross A, Macfarlane $\mathrm{P}$, et al. Prevention of coronary heart disease with pravastatin in men with hypercholesterolemia. N Engl J Med 1995; 333: 1301-7.

34. Haq IU, Jackson PR, Yeo WW, Ramsay LE. Sheffield risk and treatment table for cholesterol lowering for primary prevention of coronary heart disease. Lancet 1995; 346: 1467-71.

35. Ramsay LE, Haq IU, Jackson PR, Yeo WW, Pickin DM, Payne JN. Targeting lipid-lowering drug therapy for primary prevention of coronary disease: an updated Sheffield table. Lancet 1996; 348: 387-8.

36. Wallis EJ, Ramsay LE, Haq IU, Ghahramani P, Jackson PR, Rowland-Yeo K, et al. Coronary and cardiovascular 
risk estimation for primary prevention: validation of a new Sheffield table in the 1995 Scottish health survey population. BMJ 2000; 320: 671-6.

37. Durrington PN, Prais H, Bhatnagar D, France M, Crowley V, Khan J, et al. Indications for cholesterol-lowering medication: comparison of risk-assessment methods. Lancet 1999; 353: 278-81.

38. Fahey T. Assessing heart disease risk in primary care. BMJ 1998; 317: 1093-4

39. Isles CG, Ritchie LD, Murchie P, Norrie J. Risk assessment in primary prevention of coronary heart disease: randomised comparison of three scoring methods. BMJ 2000; 320: 690-1.

40. Greenland PH, Grundy S, Pasternak RC, Lenfant C. Problems on the Pathway from risk assessment to risk reduction. Circulation 1998; 97: 1761-2.

41. Wood D. Guidelines-a missed oportunity. Atherosclerosis 1999; 143 (Suppl 1): 7-12.

42. Plaza Pérez I, Brotons Cuixart C, Mata López P, Luque Otero M, Arnalich Fernández F, Villar Álvarez F, et al. Documentos de prevención cardiovascular: una visión global. Rev Esp Cardiol 2000; 53: 773-5. 